\title{
Bench Marking Frequent Item set Mining Models and Algorithms: Current State of the Art
}

\author{
A Muralidhar \\ Asst.Professor \\ Schooll Of Computing Sciences \& Engg. \\ VIT University, Chennai campus
}

\author{
Pattabiraman.V \\ Associate Professor \\ School Of Computing Sciences \& Engg. \\ VIT University, Chennai campus
}

\begin{abstract}
Identifying the association rules in colossal datasets is possessing elevated level of presence in data mining or data exploration. As a consequence, countless algorithms are approximated to deal alongside this issue. The two setbacks ambitious considering this outlook are: ascertaining all frequent item sets and to produce limits from them. This document is for the most portions aimed at pondering of past scrutiny, present useful rank and to ascertain the gaps of them alongside present ambitious information. Here, early subject, as it acquires extra time to process, is computationally expensive. Current discover targeted on these algorithms and their connected issues.
\end{abstract}

Keywords: Frequent Itemset Mining, utility mining, Frequent pattern mining, Association rules, Data mining

\section{INTRODUCTION}

There are a number of suggested methods for producing frequent item sets which differ in the way of spanning item set lattice, use of anti-monotone property and the way to deal with dataset. It provided in data indexing, category and clustering. Identifying frequent patterns is important in exploration organizations and connections. A substructure can be a sub-graph or sub-tree. If such substructure happens more than particular limit, it is called a regular architectural design of times. Let us talk about present position of this step such as the examined difficulties. Frequent patterns are item sets or substructures which happen in a dataset more than specified lowest no. Depending on these modifications, associate set of algorithms is explained the research differs from effective and scalable methods to most research frontiers; such as sequential, structured, correlative exploration, associative classification and frequent pattern clustering. For market basket research which examines client features from the organizations between things in the basket. In the function of association rule mining, frequent pattern mining is an important level that has been targeted at and in which amazing upgrades have been made [1].

\section{TAXONOMY}

The preliminary criteria recommended were AIS, by Agrawal et al [1]. As there are large number, Apriori seemed to be better criteria in next creation, which completely contains the infrequent itemsets. Moreover, information components for servicing are not specified. SETM, which uses SQL, is another criterion which symbolizes frequent item set by means of Apriori [1] criteria enhanced the research over frequent pattern exploration. Of information source goes also decremented of single products in information source which may type big no. Another disadvantage is that it follows tupleby-tuple strategy after every deal which is an expense. Of item sets, it is difficult to create scalable criteria for it. It uses hash tree to save reverse. [28]. Here, we recognized the design recognition techniques depending on their resemblances. In Kth successfully pass, matters of $\mathrm{K}$ item sets are acquired. But, it goes over the information source duration of lengthiest frequent item set times (n) [1] for organization concept exploration issue. With the offer of Apriori criteria, number, the issue with SETM was determined by, Agrawal and Srikant (1994) and Sarawagi et al [2]. Its disadvantage is similar to that of AIS. Of applicants which may turn into irregular later on. It is a multi-pass algorithm; where applicant item sets are established while moving the information source by increasing prior frequent item sets with each deal products. This is the draw out of the Apriori criteria [2] and its substitute [3]. Agrawal and Srikant [2] observed an exciting downwards closing property, known as Apriori which relates that products set is regular only if all its subsets are regular. But, it makes more number.

\subsection{Divergence in Itemset Mining}

Partitioning technique: It is counselled by Savasere et al [5], in that the data basis is rationally portioned into disjoint places. It needs merely 2 goes and established on statement that produce set is worldwide usual merely if it is usual at innate in at least one separated set. TID features are measured for every single set and for every single applicant in the product set.

Sampling approach: It is counselled by Toivonen et al [6] in that an exceptional example is excavated to understand usual product places. These product locations are considered as associate of real usual product places. For extra precise aftermath, it needs 1 or 2 goes above data source. It is just like Apriori in case of disadvantages.

Non level wise algorithm [7]: Here, applicants are adjusted afterward every single $\mathrm{M}$ deal, $\mathrm{M}$ is a parameter. It is several prosperously bypass criteria that are finished in 2 goes. It is nearer to assessing strategy.

Continuous Association Rule Mining Algorithm [8]: It determines usual product locations online. It permits modifying factors, lowest assistance and lowest assurance at each deal across early prosperously pass. It is a 2 prosperously bypass criteria enabling none fixed enhance of applicants. 
Hidber, 1999 [8] display that it is less able than Apriori, but has less storage use.

\subsection{Tree Models}

FP tree based algorithm: Sometimes, after Apriori criteria reduces no. of applicants unexpectedly, it encounters two nontrivial expenses. They are, growing colossal no. of applicant locations and usually advancing the data basis and assessing the applicants employing design related. Han et al. [4] counselled this criterion that decides finish set of usual product locations lacking applicant conception, reliant on tear and vanquish strategy. It rise a catalogue of usual produce in preliminary prosperously bypass and kinds them in regularity decrement way. The data basis is next compacted into FP tree. The FP tree is excavated, commencing from a usual period 1 design, growing its sub data basis (having set of prefix paths co transpiring alongside suffix pattern). This is completed iteratively. Disadvantages of FP tree are period intake, no versatility and no reusability.

This criterion separates the setback of ascertaining long usual styles into tiny styles and concatenating the suffixes and therefore cutting find time. The supplements of FP progress strategy encompass of, Detail early conception, counselled by Agarwal et al [27], H-Mine, By Pei et al [11], Top-down and Bottom-up traversals by Liu et al [12] and scope concentrated prefix tree framework by Grahne and Zhu [13].

\subsection{Interesting Item set Mining}

Sometimes it becomes vital for client to ponder merely needed styles.

Constraint-based mining: A client could be passionate concerning the styles fulfilling a little enumerated restriction. The arrangement of sports convertible constraints was additionally suggested by him [18] to more trim the data find space alongside dull constraints. Gade et al [19] suggested a stop check that decides product set's significance reliant on heavy stop instituted by pattern's items. The power of monotonic constraints was remarked by Grahne et al [15]. Bonchi et al [17] suggested double exploration. Yun and Leggett [21] suggested a heavy usual product set discovery criteria to locale heaviness check as manipulating downwards closing property $\operatorname{avg}()=v)$, is led by coordinating them in an order for restricted design progress [11]. Bonchi and Lucchese [20] suggested a criteria for discovery shut restricted styles. g. For example, helpful constraints have to be allocated at the introduction of mining; anti monotonic have to be allocated forceful to retain design progress below manipulation across discovery and monotonic constraints that need merely one check verifying [14]. Limits can be of disparate kinds reliant on their contact alongside discovery function.

\section{CURRENT STATE OF THE ART}

\subsection{Item set Mining using functional parameters or utilities}

Here in this exercise the utility is identification assess to ascertain the worth of an item set in respect to earnings. Hence a discovery strategy recognized as Request discovery can be believed to present prosperous usual item set discovery swiftly techniques. In most of the marketplace bag methods it is momentous to understand the regularity of constituents alongside respect to paycheck they produce, that frequently not discovered in dealings alongside needed support. It could additionally be an exercise of the established sketches that from time to time tremendously prosperous constituents could be removed by finishing them as irregular. The produce regularity in given dealings that these established sketches ponder are not genuine to ascertain the constituents that are usual and tremendously successful. These sketches focus on the produce by their lifestyle the deal. The established ARM methods are definitely is not able in this contest as these sketches always gives utility of every single factor as 1 and the quality of that factor is always 1 if it prevails in a given deal or 0 if not prevails Conventional methods of ARM weights comparably the all constituents encompassed in dealings of the given dataset.

It usually overestimates, especially at the starting levels, where the variety of applicants techniques the variety of all the blends of products. This is useless whenever the variety of unique products is large and application limit is low. By application exploration, several essential choices in business area like increasing income, reducing marketing or stock costs can be taken and more essential knowledge about item sets/customers causing the majority of the benefit can be found. Later, the identical writers suggested two new methods UMining and UMining $\mathrm{H}$ [29] to figure out the great usefulness The current these great application design exploration methods [28], [29], [30], [32] experience from the level-wise applicant generation- and-test problem. Therefore, they produce many applicants and need several data source tests to my own the actual great effectiveness styles. These allow the user to ideally evaluate the value of an item set by the application value. They cannot maintain the downwards closing residence of Apriori. The great application exploration designs were described in [28], [29], [30], [31] and [32]. They used a heuristic to figure out whether an item set should be considered as a candidate

Item set. Other application areas, such as scientific gene data source, web click sources, stock tickers, system traffic dimensions, web-server information, data nourishes from indicator systems and telecommunications call information can have identical alternatives. Moreover, their variety of data source tests is straight reliant on the highest possible duration of the applicant styles. The theoretical design and explanations of great application design exploration were given in [28], known as MEU (mining with predicted utility).

Ahmed et al [33] counselled a novel tree-based applicant trimming method HUC- Trim (high request applicants prune) to effectually mine outstanding request styles lacking levelwise applicant set generation-and-test. In this stare C. CTUMy own [31] counselled an criteria that is competent above Two-Phase criteria merely in heavy data basis after the minimum request check is extremely low In the subsequent data basis check out, it discovers all the 2- factor deal heavy custom item set and reliant on that produces the applicants for 3 -element deal heavy custom item sets and so on. But yet their methods are altered alongside the level-wise applicant generation-and- examine subject and needs several data basis examinations reliant on the highest probable length of the applicant styles. It uses a design progress discovery way and needs a highest probable of three data basis examinations in difference to countless data basis examinations of the continuing methods For the early data basis check out, their criteria discovers all the 1- factor deal heavy custom item set and reliant on that produces the applicants for 2-element deal 
heavy use item sets. These criteria go across from the same subject of the level-wise applicant generation-and- examine technique. The separated items removing strategy (IIDS) [32] for discovering outstanding request item sets was counselled to cut a little applicants in every single bypass of directories. They projected competent outstanding request item set discovery criteria FUM and DCG+ and exposed that their work is larger than all preceding outstanding request design discovery works. They have described "transaction heavy utilization", and shown it can uphold the rising down closing residence. F. It prunes a large number of needless applicants across the discovery process. At the last check out, it discovers out the real outstanding request item sets from outstanding deal heavy custom item sets. The Two-Phase [30] criteria was projected reliant on the explanations of [28] to find outstanding request item sets employing the downwards closing residence.

Though, their strategy cannot indicate the disparate period of lines of recency in computing the worth of dealings. Later $\mathrm{Wu}$ et al [34], Y. L. Although the sketches [28, 29, 30, 31, 32, 33, 34 , and 35] recognized below request item set discovery association, they yet drop short to completely indicate the request aspects Recency, Regularity and Commercial (RFM) in the discovery process. In the method to recency, monetary is ambitious as that the number of investment property by all clients in a design ought to drop into the scope amid the highest probable and lowest monetary limits. With the data of findings exposed above $\mathrm{Ya}-\mathrm{Han} \mathrm{Hu}$ et al [22] counselled a scoring-based method in the outlook of acting the finished believed of RFM on computing the worth of styles. In the concern of request aspect recency, the transpiring period of the last deal of a design, ought to fulfil the recency limit. In this respect they gave the RFMP-tree framework and the RFMP-growth criteria that are customized from the wellknown FP-tree framework and FP-growth criteria [36] an example of the limits noticed below request aspect "recency" is follows. The criteria for discovery styles [35] fulfilling RFM constraints; they utilized a set period gap that every single event of styles inside the given latest era of period will have the alike encounter even though of the transpiring time. Chen et al [35] remarked the quality of the data recognized as "recency" presented as request aspect that is change of data submission amid the past data and the new information.

Each node in RFMP-tree includes five fields: item-name, ranking, Fscore, Tta, parent-link, child-link, and sibling-link, whereas item-name signals up that product this node symbolizes, Rscore, Fscore, and Tta sign-up the recency ranking, regularity ranking, and finish deal number from all the dealings that have the corresponding styles displayed at this node, and parent-link, child-link, and sibling-link sign-up the features of the mother or father node, kid nodes, and the subsequent node grasping the alike item-name in the RFMPtree, respectively. Each admission in RFM-header embodies five fields: item-name, Rscore, Fscore, Tta, and go of nodelink, whereas item-name signals up that product this admission provides, Rscore, Fscore, and Tta past the recency ranking, regularity ranking, and finish deal number of this product, suitably, and go of node web link factors to the early node in RFMP-tree grasping the alike item-name. The presentation scrutiny displaying that the three limits utilized are possessing brilliant result on the counselled design. The worth of the presumed to these limits is not general. A finish RFMP-tree encompasses a past, recognized as RFM-header, and a RFMP-tree. Lastly it can be ambitious that it is vital to create generalizations the of the limits counselled and examinations demand to be gave on actual datasets that amuses disturbance and doubt e. a RFT-pattern encompassing one product only),which are categorized according to their Fscore in rising down purchase. A RFM-header is a past encompassing all the 1-RFT-patterns (i. The examinations completed on manmade datasets are validating the check principles regarded.

\subsection{Itemset Computationally-Measured Minimum Support}

Ascertaining usual item sets, all the conditions 'more or less frequent', 'high frequent' and 'completely frequent' can be thresh olds from fleecy opinions. Therefore, it is sensible to produce perhaps functional item sets in fleecy places. Users should like to enumerate a comparative minimum-support on the commonly-used era $(0,1)$ rather than on the era $(a, b)$ in the alike method, for a data basis $\mathrm{D}$, allow the assistance of item sets in $\mathrm{D}$ be allocated in an era $(\mathrm{a}, \mathrm{b})$. The phrase 'frequent' is by now a check from a fleecy outlook, recognized as the fleecy limit. In real-world data-mining plans, clients can proposal their discovery specifications in two ways:

1. This has enumerate that the key subject ought to be how to efficiently notice all usual item sets from directories lacking involve enumerating the actual minimum- assistance limit

2. 2. Definitely, clients could demand for recognizing 'more or less frequent', 'highly frequent' or 'completely frequent' item sets. This aftermath in a large gap amid the organic fleecy check and the supplies obtainable to usual item set development.

More particularly, the suggested design requires a specified limit as user-specified minimum- assistance and computationally transforms the specified limit into a real lowest assistance. When customers have mentioned their exploration specifications for regular item sets, the phrase 'frequent' is already a limit from a fluffy perspective. Shichao Zhang et al [37] suggested a new way of dealing with the minimum-support issue, made up of polynomial approximation for a specified lowest assistance on the widely used period $(0,1)$ and fluffy evaluation for a specified minimum-support in fluffy places. Shichao Zhang et al [37] have suggested a computational way of dealing with the issue of establishing the lowest assistance. Unfortunately, it is difficult to specify the minimum-support appropriate to the data source to be determined, if customers are without information concerning the data source. This allows customers to specify their exploration specifications in usually used ways, which are separate of their directories. However, even though a minimum-support is researched under the guidance of a knowledgeable miner, we cannot research whether or not the outcomes (mined with the sought after lowest support) are really what the customers want. However, current Apriori-like methods still need customers to specify the real minimum-support appropriate to the directories to be excavated. This suggested exploration technique is different from current Apriori-like methods allows customers to specify their exploration specifications in widely used ways and instantly turn the specified limit into real minimum-support (appropriate to a data source to be mined) 
This strategy needs only to know how to estimate for a given design its possibility to fulfil the restriction (this can be acquired in many situations), and it continues to be wellorganized in exercise by implementing a design space testing plan These suggestions are all based on a international systematic design, I. These writers especially concentrate on little regularity limit and how it impacts the variety of produced styles, considering set and/or proportionate limits. Another strategy has been suggested by Geerts et al. Given the present stage and the present set of regular styles, they recommend a limited of the maximum variety of applicant styles that can be produced on the next stage. Calculating the predicted variety of styles that fulfil a restriction is in common much

More difficult than estimating the possibility, that a given design meets such a restriction. [40], offering a limited higher limited on the variety of applicant styles that can occur while exploration regular styles in a level-wise establishing. In the sector of sequence exploration, [41] styles an calculate of the variety of styles due to the unique qualifications, and that are likely to be take out with regard to a regularity restriction and according to the framework of the dataset. As a result, they cannot be easily prolonged to deal with complicated conjunctions of restrictions, to incorporate different icon withdrawals or different semantics for design circumstances. e. They response the query whether there are available a regular or maximum regular item set selection that has a given variety of regular item sets of a given duration. This second issue has acquired a set of attention, resulting in many mathematical actions to evaluate the interestingness of the styles. To the best of our information, only method that has been suggested by J. [38] and [39] evaluate the possible withdrawals of regular item sets (also of shut item sets for [39] and of maximum item sets for [38]). , an exciting strategy that needs however to create complicated and particular designs. Concerning the first issue, only a few alternatives have been suggested. [38] Concentrates on the kind of withdrawals one can anticipate for various types of datasets. Besson, et al [42] to calculate the variety of styles fulfilling a restriction while preventing to create an international systematic design. [39] Determines the common variety of regular (closed) item sets using probabilistic methods.

In situation of the maximization issue this was well-known. Another query is that how fast parameter assessment can be done in other websites with identical issues as regular set exploration (exponential outcome dimension and even better look for space). Illustrations for such websites are design exploration projects with organized information like series or charts using latest outcomes from computational complexness M. Boley, et al [43] suggested that a nontrivial approximation criterion for max regular set is unlikely to be available. For 01-permanent the lifestyle of a completely polynomial randomized approximation plan has been proven in [46]. In situation of the minimization issue attempt to confirm this firmness by displaying it to be comparative to a general edition of the min set protect issue. Other essential features that can be used for parameter assessment are the variety of regular resp. shut or highest possible regular places caused by a given parameter, all of which are company keeping track of issues [44, 45]. Boley, et al [43] have examined the algorithmically projects to estimated a highest possible regular resp. It is an exciting query whether the good outcomes from processing the long lasting of a 0-1 matrix can be moved to those issues. The approximation methods have been regarded because both issues are NP- difficult. M. at least irregular set. This research is inspired by the need for an effective parameter assessment process that can be used before a probably rapid time design exploration criteria.

The restriction is to assess the highest possible assistance and lowest assistance for the regular item sets. The primary lack in randomized keeping track of is working with the calculation; the issue is recurring keeping track of process. Space state testing is concentrate on the post-processing of the information exploration process. The finding indicated for situations in which the cost of regularity keeping track of is covered with the variety of regular item sets uncomplicated randomized keeping track of generates affordable estimates in a portion of the time required for comprehensive enumeration. While in [42] the testing is not taken from information but from the design area testing. Sector professionals are required to integrate during the differential removal to set the restriction the preferred precision is based on example dimensions. However the problems in keeping track of item set are considered as NP hard issue. Applying Markov Sequence criteria in randomized keeping track of not rely on the variety of regular set but the size of the dataset. Outlined in the study [43], the MC are not good limited for common situations.

It is generally separated in three stages: 1 . the results of researched tests revealed that by lowering the lowest assistance, more exciting design can be examined. Some research on the recognition of bend is needed as a second task in regular product set criteria level; the product set matters for generated duration. In the second level the lowest assistance and series duration ' $k$ ' is identified. The suggested model is depending on testing techniques, which is a procedure to figure out lowest assistance that calculated by mean to determine possible regular item sets. The incorrect positive amount of the occurrence recognition can be reduced by: appropriate amounts of lowest assistance and duration of product set. The Apriori criteria are constructed in the information modification component. 2. Pre-processing: The pre-processing level includes 2 modules: selection and modification component. Depending on the mean running gathered outcome produce by apriori, roles of great gain are identified. Therefore, from the research an appropriate lowest assistance $40 \%$ to $50 \%$ is sufficient to generate regular item sets. Frequent product set criteria and 3. This occurs in the third level Data transformation. With the help of WSARE dataset potential features are identified in comparison with the artificial occurrence cases. Based on the result, to reduce the boring design the probable principles of lowest assistance are set at $30 \%$ to $50 \%$. In this respect Zalizah Awang Long et al [23] described a design to figure out the exciting design; lowest assistance and product set depend. Variety of product set needed for maximum recognition amount is identified on the basis of great regularity depend. The outcome of regular exploration criteria is used as an important limit in the occurrence recognition projects. The chart is examined depending on the state (increasing, reducing or stable). However approximation of values considered so still absolute principles are to be confirmed.

\subsection{Frequent Itemset Mining with Multiple Supports}

Although Wang et al. The suggested specifications are easy and effective when compared to Wang et al. A variety of exploration techniques depending on the Apriori 
specifications were suggested, each for a specific problem sector, a actual data type, or for enhancing its efficiency. Wang et al. Besides, their strategy does not consider products with quantitative principles and structured into several levels. It is thus sensible in some sense that the incident regularity of an exciting item set must be bigger than the highest possible of the smallest facilitates of the products involved in it. They suggested a bin-oriented, non-uniform assistance restriction. By the natural of products some products appear more frequently than others. Their strategy permitted customers to state different smallest facilitates to different products. In these techniques, the smallest facilitates for all the products or item sets to be huge are set at only one value. When the least assistance value of an item set is determined as the smallest lowest facilitates of the products in it, the item set may be big, but products involved in it may be little. But in authentic programs, different products may have different specifications to assess its significance. [47] Suggested a strategy for exploration organization guidelines with non-uniform smallest assistance principles. Items were arranged into dislodge places called containers, and products within the same bin were look upon as non-distinguishable with regards to the specifications of a smallest assistance. For example, assume the smallest facilitates of products A and B are set at $20 \%$ and $40 \%$ respectively under the most restriction. In this case, it is doubtable whether this item set is value considering. [48] then general the above idea and permitted the smallest assistance value of an item set to be any function of the smallest assistance principles of products in the item set. Although their strategy is soft in giving the smallest facilitates to item sets, the exploration specifications are a small complicated due to its generality. This task is, however, not always appropriate for application specifications. For the example describe above, if the assistance of product B is $30 \%$, small than its smallest assistance $40 \%$, then the 2 - item set $\{\mathrm{A}, \mathrm{B}\}$ should not be value considering. In works suggested by Y.'s strategy can fix this kind of problems, the time complexness is high. Liu et al. Lee et al [49][50], a simple specifications depending on the Apriority strategy was suggested to find the large-item sets and organization guidelines under the highest possible restriction of several smallest facilitates. The least assistance value of an item set is determined as the smallest lowest facilitates among the products in the item set. If the assistance of products is not bigger than or similar to the assistance limit, this product is not value considering. In recent literary works the fluffy huge item sets stage by stage using only one minus for the whole data source is insufficient because it cannot catch the natural and/or regularity variations of the products in the data source. As described above, the smallest assistance of products means that the incident regularity of the product must be bigger than or similar to it for being considered in the next exploration steps. Below, we will recommend well-organized specifications depending on fluffy places and Han's exploration strategy for multiple-level products to produce the fluffy huge item sets stage by stage. C.

Regular design mining is a significant data-mining technique for identifying connections among items or item sets. Due to the different wavelengths of different item sets, specifying a single lowest support cannot exactly discover exciting styles. In latest literary works the design suggested by $\mathrm{Ya}-\mathrm{Han} \mathrm{Hu}$ et al[25] is exciting, which have set a specific focus on Successive design Regular Exploration with several lowest facilitates. PLWAP-tree and PLWAP-Mine has been considered to boost because they have been confirmed to be a lightweight information framework and a highly effective criteria on sequential design mining The writers suggested that the alternatives available, which are depending on several lowest facilitates (MMS) [9] is not uncomplicated. Then, PLWAP-Mine is further comprehensive to create effective criteria, known as Multiple Supports Applicant Pattern development (MSCP-growth), for finding complete set of sequential styles with MMS. So, depending on the new explanations of sequential design with MMS, a prolonged edition of the PLWAP-tree [10] framework has been suggested, known as Preorder Connected Multiple Supports tree (PLMS-tree), to pack and store all necessary information from series directories.

To produce more exciting styles, the idea of MMS on successive design exploration and recommend criteria, known as MSCP development is particular, to discover finish set of successive design with MMS. More over the performance of the suggested PLMS-Tree framework under prolonged duration of item sets was not researched the suggested PLWAP-tree-like criteria known as PLMS-tree shops all necessary details from series information source. After that, suggested new design development criteria, known as MSCPgrowth produces the finish set of successive styles with MMS based on the PLMS-tree. The unusual product problem in the exploration of successive styles is resolved in [25]. In PLMStree we need to add some details in to the tree framework for finding finish set of successive styles whereas such is not the case with the later. The PLWAP -tree contains only products with assistance not less than min-sup while PLMS-tree maintains products with assistance not less than MIS (I). However the design described in [25] is more particular in introducing tree reflection of the information to estimate item sets, in this respect to get the scalability in storage utilization, the essential trimming process has not been mentioned.

\subsection{Mining Frequent Itemsets With no Candidature}

This landmark improvement of regular item sets exploration prevents the expensive applicant item sets creation stage, which triumphs over the primary bottlenecks of the Apriorilike methods. Highest possible duration regular item sets are produced by adjusting a design fragment development technique [55] depending on the FP-tree framework [51] suggested the FP-growth technique to avoid producing applicant item sets by building a FP-tree with only two tests over the data source. CFP-tree shops found regular item sets, but a FP-tree shops dealings. An upside down matrix strategy uses an upside down matrix to shop the dealings in a special structure, then creates and mines relatively small components, which are known as COFI-trees [53]. Some criteria example for FP-growth without producing applicant item sets is temporarily described as follows. The H-mine technique [52] uses a memory-based extremely framework to shop a rare data source in the primary storage, and creates an H-structure to entice FP-growth in exploration heavy directories. Han et al. They similarly use prefix and suffix discussing in the CFPtree, but only prefix discussing in the FP-tree. The CFP-tree framework [54] is designed to shop pre-computed regular item sets on a hard drive to obtain space.

It is unspecified that there is no restriction on the main storage space. In addition, dealings can be additional to or eliminated from the tree at any time. William Cheung et al [56] presented Compacted and Organized Deal Series Plant or CATS Plant and CATS Plant techniques. Here in this style building a 
CATS Plant helps to use for several frequent style discovery with different facilitates. The rumours is genuine for a properly huge databases due to the following reasons: 1) the current pattern of modern processing goes towards pcs with loads of main storage space (gigabytes sized); 2) storage space management techniques and information pressure strategy in the CATS Plant decrease storage space footprint; Furthermore, CATS Plant and CATS Plant techniques allow single complete frequent style discovery and transaction flow discovery.

In suggested CATS-Tree, huge item sets for different lowest supports can be found with the same information framework. Under the performance assessment, performance of tree development as well as of organization concept exploration is performed. Under the storage specifications of methods, the tests targeted on storage specifications for tree development and storage specifications for organization concept exploration This SC Tree criteria is used to make simpler the procedure of tree development and another is to make simpler the concept exploration method. That is, no renovation is needed when the lowest assistance is changed. CATS-tree allows users to adjust the lowest assistance value. In this regard Chuang-Kai Chiou et al [26] presented a new tree framework known as Categorized Pressure (SC) tree and exploration criteria for organization concept are suggested. Later, Collection Compacted tree (GC tree) was suggested by Liou et al [57] to further improve the performance of CATS tree. Advantages of several methods are combined in this criteria, and trial results also show that the suggested criteria outperforms Compacted and Arranged Deal Series tree (CATS tree) [4DM] and Collection Compacted tree (GC tree) [4DM] and Collection Compacted tree (GC tree) [57] exploration methods. As a result, the cost of organization concept exploration is reduced and it is not only more effective but also space-saving. The storage specifications for constructing and exploration the tree framework of SC tree is also less than the other methods and it is the most effective criteria. The performance of tree development and organization concept exploration is improved by SC tree. Under the experiment and assessment section the performance of SC tree, CATS tree and GC tree criteria was verified. Focus is on execution performance and storage specifications under different values of transaction size feature. By applying the SC tree criteria in huge database by employing the technique of similar and distributed processing, the scalability also increases. By pre-sorting the information set, the information arrangement of SC tree is consistent, so that powerful modification of the tree framework can be avoided. The CATS tree is one of them and it builds its tree framework dynamically so that the exploration procedure is complex and tedious. Hence, an enhanced criteria known as the Categorized Pressure tree (SC tree) where organization rules can be excavated in a bottom-up style instead of bi-directional or recursive is suggested.

Table 1: Feature exploration of the models in recent literature

\begin{tabular}{|l|l|}
\hline \multirow{3}{\text{TreeBased}}{} & {$[22],[25],[26],[31],[33],[34],[35],[51]$,} \\
\hline & {$[52],[53],[54],[55],[56],[57]$} \\
Apriori Based & {$[23],[28],[29],[30],[32],[37],[38],[39]$,} \\
\hline & {$[40],[41],[42],[43],[47],[48],[49],[50]$} \\
Candidature & {$[23],[28],[29],[30],[32],[37],[38],[39]$,} \\
\hline $\begin{array}{l}\text { support } \\
\text { constraint }\end{array}$ & {$[22],[25],[42],[43],[47],[48],[49],[50]$} \\
\hline
\end{tabular}

\begin{tabular}{|l|l|}
\hline & {$[42],[43],[47],[48],[49],[50]$} \\
\hline $\begin{array}{l}\text { User defined } \\
\text { support }\end{array}$ & {$[22],[31],[33],[34],[35],[28],[29],[30]$,} \\
\hline $\begin{array}{l}\text { computed } \\
\text { support }\end{array}$ & {$[32],[47],[38],[39],[48],[41],[42],[49],[50]$,} \\
\hline $\begin{array}{l}\text { Multiple } \\
\text { supports }\end{array}$ & {$[25],[37],[23],[40]$} \\
\hline & {$[48],[49],[50],[25],[47]$} \\
\hline & {$[22],[31],[33],[34],[35],[28],[29],[30]$,} \\
\hline & {$[32]$} \\
\hline & {$[26],[51],[52],[53],[54],[55],[56],[57]$,} \\
& {$[48],[49],[50],[25],[47],[43],[38],[39]$,} \\
& {$[41],[42],[37],[23],[40],[22],[33],[34]$,} \\
& {$[35],[28],[29],[32]$}
\end{tabular}

\section{CONCLUSION}

Much analysis is still needed to considerably reduce the size of produced design places and improve the high quality of maintained styles under consideration of the restrictions such as adjusting, temporary and inference credibility. There are suggestions on reduction of such a large set, such as shut styles, maximum styles, estimated styles, compacted design angles, associate styles, utility styles, and discriminative regular styles. We feel the bottleneck of regular design exploration is not on whether we can obtain the complete set of regular styles under certain restrictions effectively but on whether we can obtain a lightweight but top high quality set of styles that are most useful in programs. First, the most targeted and considerably analyzed subject in regular design exploration is perhaps scalable exploration techniques, which is still a significant issue that still having issues to settle for efficient exploration strategies. With numerous literary works released about regular design exploration designs that confirmed in this paper, obvious that there are still several critical analysis problems that need to be fixed in the process of regular item set exploration, which is a foundation approach in data exploration programs, in particular organization concept exploration and category. However, it is still not clear what kind of styles will give us sufficient design places in both compactness and associate high quality for a particular application, and whether we can own such styles straight and effectively. There is a big opportunity to perform analysis to recognize post item set exploration designs to figure out the consistent item sets. The set of regular styles produced by most of the current design exploration techniques is too large for effective utilization. In this respect we continue our analysis to figure out scalable shut item set exploration designs under the restrictions researched. The existing benchmarking Utility based Mining Models [28, 29, $30,32,22,31,33,34$, and 35] are

- based on user defined supports, and most of them are multi-pass models [28, 29, 30, $32,22,31,33,34,35]$

○ few of them are dual pass models[30, 31]

- None of them is proven to be compatible for multiple supports

The Benchmarking models $[23,37,38,39,40,41,42,43,48$, $49,50,25]$ that require support to find frequent items are computing the support through statistical approaches such as probability [37], sampling[23], averages[38, 39], upper 
bounds[40], estimation[41, 42], maximal constraints[49,50], pre-order links[25], bin oriented[48] and algorithmic [43]. The observations indicate that no statistical model is frequently used, hence none of these statistical approaches are not significant to consider as benchmark.

In this regard we can conclude that utility based mining with multiple supports and/or with computed supports and/or without support constraints is an optimal research direction. Considering transition state and temporal validity of the transactions in mining models defined as benchmarking [28, $29,30,32,22,31,33,34$, and 35] is another significant issue of research. In this regard we estimate our further research in directions said above.

\section{REFERENCES}

[1] Agrawal, R., T, Imielinski and A, Swami, 1993, Mining association rules between sets of items in large databases, Proceedings of the 1993 ACM SIGMOD International Conference on Management of Data, May 25-28, ACM, New York, USA., pp: 207-216

[2] Agrawal, R, and R, Srikant, 1994, Fast algorithms for mining association rules, Proceedings of the 20th International Conference on Very Large Data Bases, Sept, 12-15, San Francisco, CA., USA., pp: 487-499

[3] Mannila, H., H, Toivonen and A, Inkeri Verkamo, 1994 Efficient algorithms for discovering association rules Proceedings of the AAAI Workshop on Knowledge Discovery in Databases, (KDD-94), IEEE, pp: 181-192

[4] Han, J., J, Pei, Y, Yin and R, Mao, 2004, Mining frequent patterns without candidate generation: A frequent-pattern tree approach, Data Mining Knowledge Discovery, 8: 53-87

[5] Savasere, A., E, Omieccinski and S, Navathe, 1995, An efficient algorithm for mining association rules in large databases, Proceedings of the 21st International Conference on Very Large Databases, Sept, 11-15, Zurich, Switzerland, pp: 432-443

[6] Toivonen, H., 1996, Sampling large databases for association rules, Proceedings of 22th International Conference on Very Large Databases, Sept, 3-6, Bombay, India, pp: 134-145

[7] Brin, S., R, Motwani and C, Silverstein, 1997, Beyond market basket: Generalizing association rules to correlations, Proceedings of the 1997 ACM SIGMOD International Conference on Management of Data, May 11-15, Tucson, AZ., pp: 265-276

[8] Hidber, C., 1999, Online association rule mining, ACM SIGMOD Rec., 28: 145-156

[9] B. Liu, W. Hsu, and Y. Ma, "Mining association rules with multiple minimum supports,", Proceedings of the fifth ACM SIGKDD international conference, San Diego, CA, USA August 15-18, 1999, p.341

[10].Ezeife, C.I.; Min Chen; Incremental mining of Web sequential patterns using PLWAP tree on tolerance MinSupport, Database Engineering and Applications Symposium, 2004, Issue Date: 7-9 July 2004, On page(s): 465 - 469

[11] Pei, J., J, Han and L,V,S, Lakshmanan, 2001, Mining frequent itemsets with convertible constraints,
Proceedings of the 17th International Conference on Data Engineering, April 2-6, Heidelberg, Germany, pp: $433-$ 332

[12] Liu, J., Y, Pan, K, Wang and J, Han, 2002, Mining frequent item sets by opportunistic projection, Proceedings of the 8th ACM SIGKDD International Conference on Knowledge Discovery in Databases, July 23-26, Edmonton, Canada, pp: 239-248

[13] Grahne, G, and J, Zhu, 2003, Efficiently using prefixtrees in mining frequent itemsets, Proceedings of the 2003 ICDM International Workshop on Frequent Itemset Mining Implementations, (IWFIMI03), Melbourne, FL., pp: $123-132$

[14] Lakshmanan, L,V,S., R, Ng, J, Han and A, Pang, 1999, Optimization of constrained frequent set queries with 2variable constraints, ACM SIGMOD Rec., 28: 157-168

[15] Grahne, G., L, Lakshmanan and X, Wang, 2000, Efficient mining of constrained correlated sets, Proceedings of the 2000 International Conference on Data Engineering, Feb, 28-March 3, San Diego, CA., pp: $512-521$

[17] Bucila, C., J, Gehrke, D, Kifer and W, White, 2003, DualMiner: A dual-pruning algorithm for itemsets with constraints, Data Min, Knowl, Discov., 7: 241-272

[18] Bonchi, F., F, Giannotti, A, Mazzanti and D, Pedreschi, 2003, Exante: Anticipated data reduction in constrained pattern mining, Proceedings of the 7 th European Conference on Principles and Practice of Knowledge Discovery in Databases, Sept, 22-26, Cavtat, Dubrovnik, Croatia, pp: 59-70

[19] Gade, K., J, Wang and G, Karypis, 2004, Efficient closed pattern mining in the presence of tough block constraints, Proceedings of the 10th ACM SIGKDD International Conference on Knowledge Discovery and Data Mining, Aug, 22-25, Seattle, WA., pp: 138-147

[20] Bonchi, F, and C, Lucchese, 2004, On closed constrained frequent pattern mining, Proceedings of the 2004 International Conference on Data Mining, Nov, 1-4, Brighton, UK., pp: 35-42

[21]Yun, U, and J, Leggett, 2005, Wfim: Weighted frequent itemset mining with a weight range and a minimum weight, Proceedings of the 2005 SIAM International Conference on Data Mining, April 21-23, Newport Beach, CA., pp: 636-640

[22] Ya-Han Hu; Fan Wu; Tzu-Wei Yen "Considering RFM-values of frequent patterns in transactional databases", 2nd International Conference on Software Engineering and Data Mining (SEDM), June 2010, pages: $422-427$

[23] Long, Z.A. Hamdan, A.R. Bakar, A.A; Parameter setting procedure via quick parameter evaluation in frequent pattern mining for outbreak detection, 2nd Conference on Data Mining and Optimization, 2009. DMO '09, Issue Date: 27-28 Oct. 2009, On page(s): 90 93

[24] Antunes, C.; Pattern Mining over Star Schemas in the Onto4AR Framework, IEEE International Conference on Data Mining Workshops, 2009, ICDMW '09, Issue Date: 6-6 Dec. 2009, On page(s): 453 - 458 
[25] Ya-Han Hu; Fan Wu; Yi-Chun Liao; Sequential pattern mining with multiple minimum supports: A tree based approach, 2nd International Conference on Software Engineering and Data Mining (SEDM), Issue Date: 23-25 June 2010 On page(s): 428 - 433

[26] Chuang-Kai Chiou, Judy C. R. Tseng; Sorted Compressed Tree: An Improve Method of Frequent Patterns Mining without Support Constraint, 2nd International Conference on Software Engineering and Data Mining (SEDM), 2010, Issue Date: 23-25 June 2010, On page(s): 328 - 333

[27] Agarwal, R,C., C, Aggarwal and V,V,V, Prasad, 2001, A tree projection algorithm for generation of frequent item sets, J, Parallel Distributed Comput., 61: 350-371

[28].Yao, H., Hamilton, H.J., Butz, C.J.: A Foundational Approach to Mining Itemset Utilities from Databases. In: Third SIAM Int. Conf. on Data Mining, pp. 482-486 (2004)

[29].Yao, H., Hamilton, H.J.: Mining itemset utilities from transaction databases. Data \& Knowledge Engineering $59,603-626(2006)$

[30]. Liu, Y., Liao, W.-K., Choudhary, A.: A Two Phase algorithm for fast discovery of High Utility of Itemsets. In: Ho, T.-B., Cheung, D., Liu, H. (eds.) PAKDD 2005. LNCS(LNAI), vol. 3518, pp. 689-695. Springer, Heidelberg (2005)

[31]. Erwin, A., Gopalan, R.P., Achuthan, N.R.: CTU-Mine: An Efficient High Utility Itemset Mining Algorithm Using the Pattern Growth Approach. In: 7th IEEE Int. Conf. on Computer and Information Technology (CIT 2007), pp. 71-76 (2007)

[32]. Li, Y.-C., Yeh, J.-S., Chang, C.-C.: Isolated items discarding strategy for discovering high utility itemsets. Data \& Knowledge Engineering 64, 198-217 (2008)

[33]. Tanbeer, S.K., Ahmed, C.F., Jeong, B.-S., Lee, Y.-K.: CP-tree: A tree structure for single pass frequent pattern mining. In: Washio, T., Suzuki, E., Ting, K.M., Inokuchi, A. (eds.) PAKDD 2008. LNCS(LNAI), vol. 5012, pp. 1022-1027. Springer, Heidelberg (2008)

[34] F. Wu, Y.-S. Lee, and J.-N. Yu, "An adaptive approach for modelselection with high stability," in Proceedings of International JointConference on e-Commerce, eAdministration, e-Society, and e-Education, Bangkok, Thailand, 2008.

[35]Y.-L. Chen, M.-H. Kuo, S.-Y. Wu, and K. Tang, "Discovering recency,frequency, and monetary (RFM) sequential patterns from customers'purchasing data," Electronic Commerce Research and Applications, vol.8, pp. 241-251, 2009.

[36] J. Han, J. Pei, Y. Yin, and R. Mao, "Mining frequent patterns withoutcandidate generation: A frequent-pattern tree approach," Data Miningand Knowledge Discovery, vol. 8, pp. 53-87, 2004.

[37] S. Zhang, et al., "Computing the minimum-support for mining frequent patterns," Knowledge and Information Systems, vol. 15, no. 2, 2008, pp. 233-257

[38] G. Ramesh, W. Maniatty, and M. J. Zaki. Feasible itemset distributions in data mining: theory and application. In Proceedings ACM PODS'03, pages 284295, 2003.

[39] L. Lhote, F. Rioult, and A. Soulet. Average number of frequent (closed) patterns in bernouilli and markovian databases. In Proceedings IEEE ICDM'05, pages 713716, 2005.

[40] F. Geerts, B. Goethals, and J. V. den Bussche. Tight upper bounds on the number of candidate patterns. ACM Trans. on Database Systems, 30(2):333-363, 2005.

[41] U. Keich and P. A. Pevzner. Subtle motifs: defining the limits of motif finding algorithms. Bioinformatics, 18(10):1382-1390, 2002.

[42] J. Besson, et al., "Parameter Tuning for Differential Mining of String Patterns," IEEE Computer Society Washington, DC, USA, 2008, pp. 77-86

[43] M. Boley, et al., "A Randomized Approach for Approximating the Number of Frequent Sets," IEEE Computer Society Washington, DC, USA, 2008, pp. 4352 .

[44] Valiant, L.G.: The complexity of computing the permanent. Theor. Comput. Sci. 8, 189-201 (1979)

[45]. Gunopulos, D., Khardon, R., Mannila, H., Saluja, S., Toivonen, H., Sharm, R.S.: Discovering all most specific sentences. ACM Trans. Database Syst. 28(2), 140-174 (2003)

[46]. Jerrum, M., Sinclair, A.: Approximating the permanent. SIAM J. Comput. 18(6), 1149-1178 (1989)

[47] B. Liu, W. Hsu, and Y. Ma, "Mining association rules with multiple minimum supports," in Proceedings of the 1999 International Conference on Knowledge Discovery and Data Mining, pp. 337-341, 1999.

[48] Y. Lee, T. Hong, and W. Lin, "Mining association rules with multiple minimum supports using maximum constraints," International Journal of Approximate Reasoning, vol. 40, pp.44-54, 2005.

[49] Y. C. Lee, T. P. Hong and W. Y. Lin, "Mining fuzzy association rules with multiple minimum supports using maximum constraints", The Eighth International Conference on Knowledge-Based Intelligent Information and Engineering Systems, 2004, Lecture Notes in Computer Science, Vol. 3214, pp. 1283-1290, 2004.

[50] Y. C. Lee, T. P. Hong and W. Y. Lin, "Mining association rules with multiple minimum supports using maximum constraints," International Journal of Approximate Reasoning, Vol. 40, No. 1, pp. 44-54, 2005.

[51] J. Han, J. Pei, Y. Yin, Mining frequent patterns without candidate generation, in: Proceedings of the 19th ACM SIGMOD International Conference on Management of Data, 2000, pp. 1-12.

[52] J. Pei, J. Han, H. Lu, S. Nishio, S. Tang, D. Yang, Hmine: hyper-structure mining of frequent patterns in large database, in: Proceedings of the 2001 IEEE International Conference on Data Mining, San Jose, CA, 2001, pp. 441-448.

[53] M. EI-Hajj, O.R. Zaiane, Inverted matrix: efficient discovery of frequent items in large datasets in the context of interactive mining, in: The ninth ACM 
SIGKDD International Conference on Knowledge Discovery and Data Mining, 2003, pp. 109-118.

[54] G. Liu, H. Lu, J.X. Yu, CFP-tree: a compact disk-based structure for storing and querying frequent itemsets, Information Sciences 32 (2007) 295-319.

[55] T. Hu, S.Y. Sung, H. Xiong, Q. Fu, Discovery of maximum length frequent itemsets, Information Sciences 178 (2008) 68-87.
[56] W. Cheung and O.R. Zaiane, "Incremental mining of frequent patterns without candidate generation or support constraint," Citeseer, 2003, pp. 111-116.

[57] S.Y. Liu, "An Efficiency Incremental Mining with Grouping Compress Tree," Unpublished master's thesis, National Central University Taoyuan Country, Taiwan, 2004 